\title{
On the simultaneous optical and near-infrared variability of pre-main sequence stars
}

C. Eiroa $^{1}$, R. D. Oudmaijer ${ }^{2}$, J. K. Davies ${ }^{3}$, D. de Winter ${ }^{4}$, F. Garzón ${ }^{5}$, J. Palacios ${ }^{1}$, A. Alberdi ${ }^{6}$, R. Ferlet ${ }^{7}$, C. A. Grady ${ }^{8}$, A. Cameron ${ }^{9}$, H. J. Deeg ${ }^{5}$, A. W. Harris ${ }^{10}$, K. Horne ${ }^{9}$, B. Merín ${ }^{11}$, L. F. Miranda ${ }^{6}$, B. Montesinos ${ }^{11}$, A. Mora ${ }^{1}$, A. Penny ${ }^{12}$, A. Quirrenbach ${ }^{13}$, H. Rauer ${ }^{10}$, J. Schneider ${ }^{14}$, E. Solano ${ }^{11}$, Y. Tsapras ${ }^{9}$, and P. R. Wesselius ${ }^{15}$

1 Dpto. Física Teórica, C-XI, Facultad de Ciencias, Universidad Autónoma de Madrid, Cantoblanco, 28049 Madrid, Spain

2 Department of Physics and Astronomy, University of Leeds, Leeds LS2 9JT, UK

3 Astronomy Technology Centre, Royal Observatory, Blackford Hill, Edinburgh, UK

4 TNO/TPD-Space Instrumentation, Stieltjesweg 1, PO Box 155, 2600 AD Delft, The Netherlands

5 Instituto de Astrofísica de Canarias, 38200 La Laguna, Tenerife, Spain

${ }^{6}$ Instituto de Astrofísica de Andalucía, Apartado de Correos 3004, 18080 Granada, Spain

7 CNRS, Institut d'Astrophysique de Paris, 98bis Bd. Arago, 75014 Paris, France

8 NOAO/STIS, Goddard Space Flight Center, Code 681, NASA/GSFC, Greenbelt, MD 20771, USA

9 Physics \& Astronomy, University of St. Andrews, North Haugh, St. Andrews KY16 9SS Scotland, UK

10 DLR Department of Planetary Exploration, Rutherfordstrasse 2, 12489 Berlin, Germany

11 L.A.E.F.F., VILSPA, Apartado de Correos 50727, 28080 Madrid, Spain

12 Rutherford Appleton Laboratory, Didcot, Oxfordshire OX11 0QX, UK

13 Department of Physics, Center for Astrophysics and Space Sciences, University of California San Diego, Mail Code 0424, La Jolla, CA 92093-0424, USA

14 Observatoire de Paris, 92195 Meudon, France

15 SRON, Universiteitscomplex "Zernike", Landleven 12, PO Box 800, 9700 AV Groningen, The Netherlands

Received 27 November 2001 / Accepted 17 January 2002

\begin{abstract}
For a complete understanding of the physical processes causing the photometric variability of pre-main sequence systems, simultaneous optical and near-IR observations are required to disentangle the emission from the stars and that from their associated circumstellar disks. Data of this sort are extremely rare and little systematic work has been reported to date. The work presented in this paper is a systematic attempt in this direction. It presents an analysis of the simultaneous optical and near-IR photometric variability of $18 \mathrm{Herbig} \mathrm{Ae} / \mathrm{Be}$ and $\mathrm{T}$ Tauri stars which were observed in October 98 by the EXPORT collaboration. The time difference between the $U B V R I$ and $J H K$ measurements is less than 1 hour in $\approx 50 \%$ of the data and the largest difference is around 2 hours in only $\approx 10 \%$ of the data. Twelve stars appear to show a correlation between the optical and near-IR variability trends, which suggests a common physical origin such as spots and/or variable extinction. The optical and near-IR variability is uncorrelated in the rest of the objects, which suggests it originates in distinctly different regions. In general, the optical variability qualitatively follows the predictions of starspots or variable extinction. As far as the near-IR is concerned, the simultaneity of the observations demonstrates that for most objects the flux is largely produced by their circumstellar disks and, consequently, in many cases the near-IR fluctuations must be attributed to structural variations of such disks producing variations of their thermal emission and/or scattered light. The observed near-IR changes of up to around $1 \mathrm{mag}$ on timescales of 1-2 days provide interesting challenges for understanding the mechanisms generating such remarkable variabilities, an issue insufficiently investigated until now but one which deserves further theoretical and modeling efforts.
\end{abstract}

Key words. stars: formation - stars: pre-main-sequence - stars: variables: general - stars: circumstellar matter accretion, accretion disks

Send offprint requests to: C. Eiroa,

e-mail: carlos.eiroa@uam.es

\section{Introduction}

Light variations have become a defining characteristic of pre-main sequence (PMS) stars. Photo-polarimetric and 
spectroscopic variability is observed towards low mass, T Tauri-type (CTTs and WTTs), and intermediate mass, Herbig AeBe-type (HAeBe), pre-main sequence (PMS) stars (e.g. Appenzeller \& Mundt 1989; Thé et al. 1994). Optical photometric variability is the best studied variable phenomenon. Regular/periodic and irregular/nonperiodic light curves are observed. Broadly speaking, three main types of optical photometric variability are distinguished (Herbst \& Shevchenko 1999):

type I: Periodic variability, mainly observed in WTTs, attributed to rotational modulation caused by cold magnetic starspots, analogous to sunspots;

type II: Irregular variations in CTTs caused by hot spots. The hot spots possibly occur at the location where material accreting along magnetic field lines from a circumstellar disk impacts onto the stellar surface. The combination of cold and hot spots explains many of the observational properties in CTTs;

type III: Irregular fluctuating light curves in addition to large amplitude non-periodic Algol-type variability, in which there is no evidence of spectral type variations or veiling. This variability is mainly observed in HAeBe stars and early type, F and G, T Tauri stars (ETTs), but also in some CTTs (BM And, Walker 1980; AA Tau, Bouvier et al. 1999; DF Tau, Chelli et al. 1999). Type III variability, also referred to as UXOR variability after the star UX Ori, is the least well understood and is attributed to variable obscuration caused by dust clumps or clouds located in a circumstellar disk and passing in front of the star (e.g. Grinin 2000). Herbst \& Shevchenko (1999) pointed out some of the merits and shortcomings of the variable obscuration model and suggested variable accretion as an alternative to explain type III variables. The variable obscuration scenario has been modeled in terms of a star + disk system, seen almost edge-on, with dust clumps screening the stellar light (Natta \& Whitney 2000, and references therein); Bertout (2000) explores type III variability by means of an accretion disk in which reprocessing of stellar and accretion luminosity leads to a flaring of the disk.

Young stars also vary in the near-IR, though relatively little systematic monitoring work has been conducted in this range and, to our knowledge, it has been limited to T Tauri stars (Skrutskie et al. 1996, the analysis of the 2MASS survey by Carpenter et al. 2001). Variability in the near-IR could be due to the phenomena indicated above for visible light, i.e. spots and variable obscuration, which mainly affect the photospheric light of the young stars. In addition, many PMS stars show near-IR excesses, which in CTTs are ascribed to accretion disks possibly with inner holes (Meyer et al. 1997). In intermediate mass stars, the origin of the near-IR excess is a long standing, poorly understood issue (e.g. Hartmann et al. 1993). Natta et al. (2001) have recently addressed it again and suggest that the excess is produced by dust evaporation in the inner puffed-up walls of a flared, optically thick disk. Thus, a further variability mechanism operating in the near-IR could be related to variable accretion leading to changes in the disk accretion luminosity and also to changes in the disk structure (Skrutskie et al. 1996; Carpenter et al. 2001). These open points emphasize the need of a more detailed characterization of the near-IR variability in HAeBe stars as they provide important constraints on the source of the near-IR emission in those stars.

In addition to the need for more systematic near-IR monitoring, in particular for the more massive HAeBe and ETT stars and especially the UXOR variables, there is a lack of simultaneous optical and near-IR observations. Data of this sort are urgently needed to constrain models addressing the causes of the PMS variability (Natta \& Whitney 2000). Simultaneous optical and near-IR photometric monitoring can reveal important clues to the variability mechanisms acting in PMS systems in the different spectral ranges. In particular, they can reveal whether the optical and near-IR variability is produced by the same physical phenomenon or whether they result from distinct mechanisms that affect the observed radiation from the star and the disk in different ways.

Observational work combining these observational techniques is extremely scarce; see Cohen \& Schwartz (1976), Rydgren \& Vrba (1983), Evans et al. (1989), Gahm et al. (1989), Hutchinson et al. (1989, 1994), Davies et al. (1990). In this context, the EXPORT observations (Eiroa et al. 2000) represent a very significant step in the right direction since simultaneous optical and near-IR photometry was collected for a large sample of PMS stars. Though our data only span for a period of time which is short compared with the characteristic variability time of these stars, their simultaneity reveals that these objects can behave very differently. In this paper, these EXPORT data are analysed and discussed.

The paper is organized as follows. In Sect. 2 we present the data drawn from our EXPORT observations. Section 3 compares the observed light curves with variability models and discusses the observed variability trends. Section 4 mainly discusses the relative contribution of the PMS photospheres and their associated circumstellar disks to the observed near-IR fluxes and the variability in this range. Finally, Sect. 5 provides some concluding remarks.

\section{Database}

\subsection{Observations}

EXPORT collected simultaneous spectroscopic and photopolarimetric data of a large sample of PMS stars (Eiroa et al. 2000). The sample includes low and intermediate mass stars, including many with UXOR variability. The primary goal was to search, on the basis of a statistically significant data set, for correlations between photopolarimetric variability and transient, $\beta$ Pic-like spectroscopic events, which are observed in many PMS stars, particularly in UXORs. By analogy to $\beta$ Pic, these spectroscopic events have been interpreted in terms of solid bodies embedded in the circumstellar disks and orbiting the stars (Grady et al. 2000 for a recent review, but see Natta et al. 2000; Beust et al. 2001). 
Table 1. PMS stars observed during October 1998 with at least two simultaneous optical and near-IR measurements. Entries in Col. 2 mean the following: H - HAeBe star, E -ETT star, C - CTT star, U - UXOR-type star. Spectral types are given in Col. 3. Column 4 provides the known variability range of the stars in the $V$ band (references are given in Col. 5). Columns 6 to 8 provide the $V, J$ and $K$ ranges as observed by EXPORT in October 98; numbers in brackets mean the number of individual measurements, each of them taken in different nights. Non-variable stars during this period are given in the last five rows of the table.

\begin{tabular}{|c|c|c|c|c|c|c|c|}
\hline Object & Class & Sp.T. & $V$-range & Ref. & $(V \text {-range })_{\text {Export }}$ & $(J \text {-range })_{\text {Export }}$ & $(K \text {-range })_{\text {Export }}$ \\
\hline & & & Variable & stars & & & \\
\hline VX Cas & $\mathrm{H}, \mathrm{U}$ & A0Vep & $10.5-13.3$ & 2 & $11.39-11.52(5)$ & $10.00-10.13(4)$ & $8.14-8.21(4)$ \\
\hline XY Per & $\mathrm{H}$ & A2IV & $9.0-10.1$ & 3 & $9.05-9.23(3)$ & 7.59-7.64 (4) & $5.97-6.00(4)$ \\
\hline CW Tau & $\mathrm{C}, \mathrm{U}$ & $\mathrm{K} 3 \mathrm{Ve}$ & $12.4-14.4$ & 1 & $12.74-14.01(5)$ & 8.94-9.37 (4) & $7.71-7.96(4)$ \\
\hline RY Tau & $\mathrm{E}, \mathrm{U}$ & F8IIIev & $9.4-11.6$ & 1 & $10.47-10.54(5)$ & $7.23-7.44(4)$ & $5.34-5.55(4)$ \\
\hline DK Tau & $\mathrm{C}, \mathrm{U}$ & $\mathrm{K} 5 \mathrm{Ve}$ & $11.7-13.9$ & 1 & $12.08-12.90(4)$ & 8.69-9.09 (4) & $6.87-7.18$ \\
\hline DR Tau & $\mathrm{C}$ & $\mathrm{K} 5 \mathrm{Vev}$ & $10.6-13.2$ & 1 & $11.78-12.34(4)$ & $8.79-9.19$ (4) & $6.76-7.12(4)$ \\
\hline UX Ori & $\mathrm{H}, \mathrm{U}$ & $\mathrm{A} 4 \mathrm{IVe}$ & $9.2-12.3$ & 2 & $9.82-10.02(5)$ & $8.76-8.92(4)$ & $7.26-7.37(4)$ \\
\hline CO Ori & $\mathrm{E}, \mathrm{U}$ & F7Vev & $9.8-12.5$ & 1 & $11.13-11.74(5)$ & $8.36-8.65(4)$ & $6.62-6.75(4)$ \\
\hline HK Ori & $\mathrm{E}, \mathrm{U}$ & G1V & $11.4-12.2$ & 3 & $11.59-11.79(5)$ & $9.31-9.34(4)$ & $7.21-7.25(4)$ \\
\hline RY Ori & $\mathrm{E}, \mathrm{U}$ & F6Vev & $11.2-13.0$ & 2 & $11.36-12.34(4)$ & $9.51-10.61(3)$ & $8.29-9.09(3)$ \\
\hline CQ Tau & $\mathrm{E}, \mathrm{U}$ & F5IVe & $9.4-11.6$ & 2 & $8.98-9.45(4)$ & $7.76-8.04(3)$ & $6.30-6.42(3)$ \\
\hline T Ori & $\mathrm{H}, \mathrm{U}$ & A3IVev & $8.7-12.6$ & 2 & $10.52-10.82(4)$ & $8.37-8.52(3)$ & $6.30-6.40(3)$ \\
\hline BF Ori & $\mathrm{H}, \mathrm{U}$ & A2IVev & $9.6-13.5$ & 2 & $9.65-9.79(4)$ & $8.92-9.03(3)$ & 7.81-7.91 (3) \\
\hline RR Tau & $\mathrm{H}, \mathrm{U}$ & A0IVev & $10.2-14.2$ & 2 & $10.94-11.46(4)$ & $9.05-9.24(3)$ & $7.00-7.08$ \\
\hline V350 Ori & $\mathrm{H}, \mathrm{U} ?$ & A2IVe & $10.7-12.5$ & 2 & $11.76-11.91(3)$ & $9.88-10.04(3)$ & $8.28-8.38(3)$ \\
\hline VV Ser & $\mathrm{H}, \mathrm{U}$ & A0Vevp & $11.1-13.3$ & 2 & $11.82-11.89(4)$ & $8.72-8.91(4)$ & $6.31-6.43(4)$ \\
\hline WW Vul & $\mathrm{H}, \mathrm{U}$ & A2IVe & $10.3-12.4$ & 3 & $10.64-11.03(5)$ & $9.23-9.37(4)$ & $7.44-7.50$ \\
\hline \multirow[t]{2}{*}{ BM And } & $\mathrm{C}, \mathrm{U}$ & $\mathrm{K} 5 \mathrm{Ve}$ & $12.4-14.6$ & 5 & $12.27-12.98(5)$ & $9.75-10.13(4)$ & $8.38-8.51(4)$ \\
\hline & & & Non-variable & stars & & & \\
\hline HD 31648 & $\mathrm{H}$ & A5V3 & 7.7 & 2 & $7.62-7.70(5)$ & $6.95-6.99(4)$ & $5.66-5.69(4)$ \\
\hline HD 34282 & $\mathrm{H}$ & $\mathrm{A} 3 \mathrm{Ve}$ & $9.7-11.2$ & 2 & $10.02-10.03(2)$ & $9.06-9.16(4)$ & $7.60-7.65(4)$ \\
\hline V346 Ori & $\mathrm{H}$ & A2IV & $9.8-11.8$ & 2 & $10.18-10.20(2)$ & $9.38-9.42(4)$ & $8.27-8.31$ \\
\hline NV Ori & $\mathrm{E}, \mathrm{U} ?$ & F6IIIe & $9.6-10.0$ & 4 & $9.78-9.81(4)$ & $8.69-8.71(3)$ & $7.61-7.64(3)$ \\
\hline PX Vul & $\mathrm{E}, \mathrm{U}$ & F3Ve & $11.5-11.8$ & 2 & $11.51-11.55(5)$ & $9.30-9.36(4)$ & $7.77-7.85(4)$ \\
\hline
\end{tabular}

Ref.: (1) Herbst et al. (1994), (2) Thé et al. (1994), (3) Shevchenko et al. (1993), (4) de Winter et al. (2001), (5) Grinin et al. (1995).

The observations were carried out during four periods of around 4 days each. Details of the observing runs, instrumental setup and results are presented by Eiroa et al. (2001, near-IR photometry), Mora et al. (2001, spectroscopy) and Oudmaijer et al. (2001, optical polarimetry and photometry). Photometric errors are smaller than $0.05 \mathrm{mag}$ on most of the individual measurements (see Eiroa et al. 2001; Oudmaijer et al. 2001); thus, the photometry data quality is good enough to distinguish photometric variations at the $0.1-0.15 \mathrm{mag}$ level, and even better when the simultaneous or quasi-simultaneous character of the photometry in 8 photometric bands, $U B V R I$ and $J H K$, is considered. Most of the PMS stars of the sample showed spectroscopic and photo-polarimetric long term (months) and short term (days, hours) variabilities. For an analysis of the simultaneous near-IR and optical photometric variability, the best period was October 98 when we were able to obtain sufficient simultaneous data of a significant number of stars. The present paper is restricted to data collected during this run since it is aimed at assessing whether the optical and near-IR variability can be ascribed to the same or to different physical mechanisms operating at the same time in PMS systems.

\subsection{The sample}

Twenty three PMS stars were observed simultaneously during October 98 with at least two measurements in both optical and near-IR ranges, each measurement taken in different nights. Most of the stars were observed in four different nights. The time difference between the $U B V R I$ and $J H K$ observations is less than one hour for $\approx 50 \%$ of the measurements; the largest difference is around 2 hours, but for only $\approx 10 \%$ of the data.

Individual $U B V R I J H K$ values can be found in Eiroa et al. (2001, JHK magnitudes) and Oudmaijer et al. (2001, UBVRI magnitudes). The number of data points per star and photometric band ranges from 2 to 5 . The stars are listed in Table 1. UXOR-type stars are classified on the 
basis of the optical light curves and polarimetry (Oudmaijer et al. 2001). Spectral types are taken from Mora et al. (2001). The stars HD 31648, HD 34282, NV Ori, V346 Ori and PX Vul remained constant; for these objects the largest peak-to-peak variation is less than $0.1 \mathrm{mag}$ and most peak-to-peak variations are less than 0.05 mag. The remaining 18 stars were definitely variable during this run, i.e. $\Delta m>0.15 \mathrm{mag}$ in at least two different photometric bands. We note that in Table 1 most of the variable stars (16 out of 18 ) are identified as UXORs; 9 stars have spectral type A, 4 are F type, 1 is $\mathrm{G}$ type and 4 are $\mathrm{K}$ type; this result is most likely due to the bias introduced by compiling the observed EXPORT sample rather than an intrinsic effect. We also note that 11 out of 18 were close to their known maximum brightness or above the known mean $V$ magnitude (VX Cas, XY Per, DK Tau, UX Ori, HK Ori, CQ Tau, BF Ori, RR Tau, VV Ser, WW Vul, BM And), 3 stars were at average brightness (DR Tau, T Ori, V350 Ori) and 4 stars were below the average level, at least during some individual measurements (RY Tau, CW Tau, CO Ori, RY Ori).

\section{Light variations of the stars}

The most significant aspect of our data is the feasibility of performing a comparison of the optical and near-IR variations and thus assessing whether the simultaneous behaviour in both wavelength regimes has the same origin or has distinct physical causes. To our knowledge, such an analysis, which is highly relevant for theoretical models dealing with these issues (e.g. Natta \& Whitney 2000), has not yet been performed.

As a first approach, we can assume that the optical and near-IR variations are caused by the same single physical mechanism in those stars with the same variability trend in both wavelength regimes. That is to say, the stars become simultaneously brighter or fainter in the optical and in the near-IR. On the other hand, we can further assume that the optical and near-IR variabilities are caused by different physical processes in those stars not showing a correlation of their optical and near-IR brightness variations, i.e. they show different variability trends.

In the next subsection we carry out a general comparison of the observed light variations with the predictions of standard PMS variability models, while the stars are classified in Sects. 3.2 and 3.3 according to the observed (anti-)correlation of their optical and near-IR variations.

\subsection{Comparison with variability scenarios}

A rough analysis of the data can be made in terms of the variability scenarios referred to in the Introduction, i.e. spots and variable obscuration. In order to carry out this analysis we follow the procedure by Fernández \& Eiroa (1996), which is similar to other schemes (see references therein, and Carpenter et al. 2001 for a recent discussion). For spots, the procedure assumes that the star and the spot radiate as black bodies. The stellar temperature and the observed $V$-brightness variability amplitude are used as the main input parameters, while the stellar surface covered by the spot and its temperature are free parameters. These spot empirical models provide ranges in area and temperature of the star spots, which predict brightness variability amplitudes at the different wavelengths. For variable obscuration, the variability is produced by dust clouds or clumps in an inhomogeneous circumstellar envelope or disk passing in front of the star. The variability amplitudes follow an extinction law according to the size and nature of the dust particles and, initially, a comparison with a normal interstellar extinction law can be made (e.g., Cohen et al. 1981). All models predict overall light curves and also wavelength-dependent brightness variability, as already pointed out. This latter aspect is particularly relevant for our case and is what we use in fact, since our limited number of data points does not recover complete light curves. Thus, we have to further point out that the predicted amplitudes are "normalized" to the $V$ amplitude introduced as an input parameter. It is also important to note that both spots and variable obscuration predict photometric variations with amplitudes which decrease with wavelength. More details are given by Fernández \& Eiroa (1996).

Table 2 compares the amplitude of the observed simultaneous variability with the expected amplitudes produced by spots and variable obscuration in BM And, CW Tau, DK Tau and DR Tau, all the CTTs in Table 1. Only some illustrative solutions of the possible range of spot parameters are shown. We note that a detailed comparison between our data and model predictions cannot be made because of the limited number of data points and the fact that a choice among spot solutions and variable extinction cannot always be taken. Nevertheless, trends can be roughly distinguished and exotic solutions can be excluded. For example spots covering more than $50 \%$ of the star surface or variable obscuration producing more nearIR than optical extinction can be ruled out. The results in Table 2 suggest that the variability amplitude predictions of variable extinction, assuming a standard interstellar extinction law, tend to differ from the observed ones - particularly the observed near-IR variations are larger than the predicted ones. If variable obscuration is the mechanism at work, this suggests that the dust grains causing the variability are larger than normal interstellar grains. In the case of spots, for some stars, BM And and DK Tau in Table 2, spot model predictions agree reasonably well with the observed optical and near-IR brightness amplitudes (the observed amplitudes are better reproduced by cold spots in the case of DK Tau with the exception of the $U$ amplitude). The observed $U$ variability in CW Tau is definitely smaller than the values predicted by any kind of spots. The observed near-IR amplitudes of this star seem to be smaller than expected from cold spot models while they appear larger in the case of DR Tau. These results could reflect the relative contribution of the stellar photosphere and the disk, in particular to the near-IR fluxes (see Discussion below). We do not perform this spot 
Table 2. Comparison of observed variability amplitudes with the predictions of spot and variable obscuration models. Below the star's names the adopted effective temperatures are given. Area and temperature of the selected spot solution (among a large range of possible spot parameters) are shown in Col. 2. Observed amplitudes are given in the first row of the part of the table corresponding to each star, while the predicted spot amplitudes are given in the subsequent rows; the last row corresponds to variable extinction predictions. The minus sign in the hot spot solutions means that star+spot is brighter than the star alone.

\begin{tabular}{|c|c|c|c|c|c|c|c|c|c|}
\hline $\begin{array}{l}\text { Object } \\
T_{*}(\mathrm{~K})\end{array}$ & $\begin{array}{l}\text { Observed } \\
\operatorname{Spot}\left(T_{\mathrm{s}}(K), \text { Area }\right) \\
\text { Variable Extinction }\end{array}$ & $\Delta U$ & $\Delta B$ & $\Delta V$ & $\Delta R$ & $\Delta I$ & $\Delta J$ & $\Delta H$ & $\Delta K$ \\
\hline \multirow{4}{*}{$\begin{array}{l}\text { BM And } \\
(4400)\end{array}$} & Observed & .95 & .80 & .68 & .60 & .51 & .38 & .20 & .13 \\
\hline & $(3800,0.31)$ & .86 & .85 & .71 & .62 & .51 & .34 & .28 & .23 \\
\hline & $(4800,0.47)$ & -1.04 & -.83 & -.65 & -.55 & -.44 & -.28 & -.23 & -.19 \\
\hline & Var. Extinc. & 1.04 & .89 & .68 & .56 & .41 & .18 & .11 & .06 \\
\hline \multirow{5}{*}{$\begin{array}{l}\text { CW Tau } \\
(4700)\end{array}$} & Observed & .76 & 1.05 & 1.02 & .90 & .74 & .41 & .25 & .17 \\
\hline & $(3200,0.28)$ & 1.05 & 1.09 & .99 & .90 & .80 & .62 & .52 & .45 \\
\hline & $(3800,0.36)$ & 1.22 & 1.18 & 1.00 & .87 & .74 & .51 & .43 & .36 \\
\hline & $(5600,0.28)$ & -1.79 & -1.43 & -1.06 & -.85 & -.66 & -.40 & -.32 & -.26 \\
\hline & Var. Extinc. & 1.56 & 1.34 & 1.02 & .84 & .62 & .27 & .16 & .09 \\
\hline \multirow{4}{*}{$\begin{array}{l}\text { DK Tau } \\
(4400)\end{array}$} & Observed & .95 & .56 & .48 & .46 & .48 & .40 & .37 & .31 \\
\hline & $(2800,0.14)$ & .47 & .53 & .50 & .46 & .42 & .35 & .30 & .26 \\
\hline & $(5200,0.11)$ & -.86 & -.69 & -.50 & -.40 & -.29 & -.10 & -.07 & -.06 \\
\hline & Var. Extinc. & .73 & .63 & .48 & .39 & .29 & .13 & .07 & .04 \\
\hline \multirow{4}{*}{$\begin{array}{l}\text { DR Tau } \\
(4400)\end{array}$} & Observed & .64 & .69 & .56 & .48 & .39 & .40 & .39 & .36 \\
\hline & $(3600,0.21)$ & .66 & .68 & .59 & .52 & .43 & .30 & .24 & .21 \\
\hline & $(5200,0.12)$ & -.92 & -.75 & -.54 & -.42 & -.31 & -.18 & -.14 & -.12 \\
\hline & Var. Extinc. & .86 & .73 & .56 & .42 & .34 & .15 & .09 & .05 \\
\hline \multirow{6}{*}{$\begin{array}{l}\text { CQ Tau } \\
(6650)\end{array}$} & Observed & .79 & .57 & .46 & .39 & .31 & .28 & .19 & .11 \\
\hline & $(5400,0.24)$ & .62 & .56 & .47 & .41 & .35 & .25 & .22 & .19 \\
\hline & $(5600,0.28$ & .67 & .59 & .49 & .42 & .36 & .26 & .22 & .19 \\
\hline & $(7200,0.44)$ & -.67 & -.54 & -.42 & -.36 & -.29 & -.20 & -.17 & -.15 \\
\hline & $(7400,0.33)$ & -.73 & -.58 & -.44 & -.38 & -.30 & -.21 & -.18 & -.15 \\
\hline & Var. Extinc. & .70 & .60 & .46 & .38 & .28 & .12 & .07 & .04 \\
\hline
\end{tabular}

model exercise for the HAeBe and ETT stars of Table 1 because starspot models are most likely inappropriate for them (e.g. Herbst et al. 1994; Herbst \& Shevchenko 1999). Nevertheless, the stars of these types showing a time correlation of the optical and near-IR behaviour (see next section) have variability amplitudes which could formally be fit by simple spot models (or a variable extinction scenario). As an example of this, some spot parameter results fitting the observations of the ETT star CQ Tau are shown in Table 2, but see below for more details on this star.

\subsection{Stars with similar optical and near-IR variability trends}

In this subsection we discuss the objects that behave similarly in the optical and near-IR, i.e. they either brighten or fade simultaneously.

VX Cas, XY Per, HK Ori, T Ori, BF Ori, RR Tau, V350 Ori, VV Ser, WW Vul, BM And: These stars were observed close to maximum or above the average brightness level. The observed variability was relatively small in all photometric bands, $\Delta V<0.4$, $\Delta J<0.19, \Delta K<0.12$ (XY Per and HK Ori remained constant in the near-IR); the exception was BM And with
$\Delta V=0.71, \Delta J=0.38 \Delta K=0.13$. The optical and nearIR photometric light curves follow the same trend in time, thus giving us confidence in the very small variations observed in some cases. As an illustration, Fig. 1 shows the data for BM And and VX Cas. The brightness changes decrease with increasing wavelength; the color changes are small and tend to be either redder when the stars are fainter or close to constant. This behaviour can be explained in terms of spots and/or variable extinction; thus, in the first instance, a single scenario could account for the simultaneous optical and near-IR light curves in each star, although the precise mechanism can vary from star to star.

CW Tau, DR Tau: The variability of CW Tau was large from JD 2451110.5 to 2451111.5 , and from that date to 2451114.5 it was smaller, staying close to its known minimum brightness (Fig. 2). The amplitudes decrease with wavelength from $V$ to $K$. The $B$ and $V$ amplitudes are similar and slightly larger than the $U$ amplitude. $U$ is systematically brighter than $B, U-B<0.0$, which departs significantly from its known average $U-B=0.20$ (Herbst et al. 1994; Bouvier et al. 1995). For the $V-R$ to $H-K$ colors, the star reddens when it becomes fainter; $B-V$ remains 

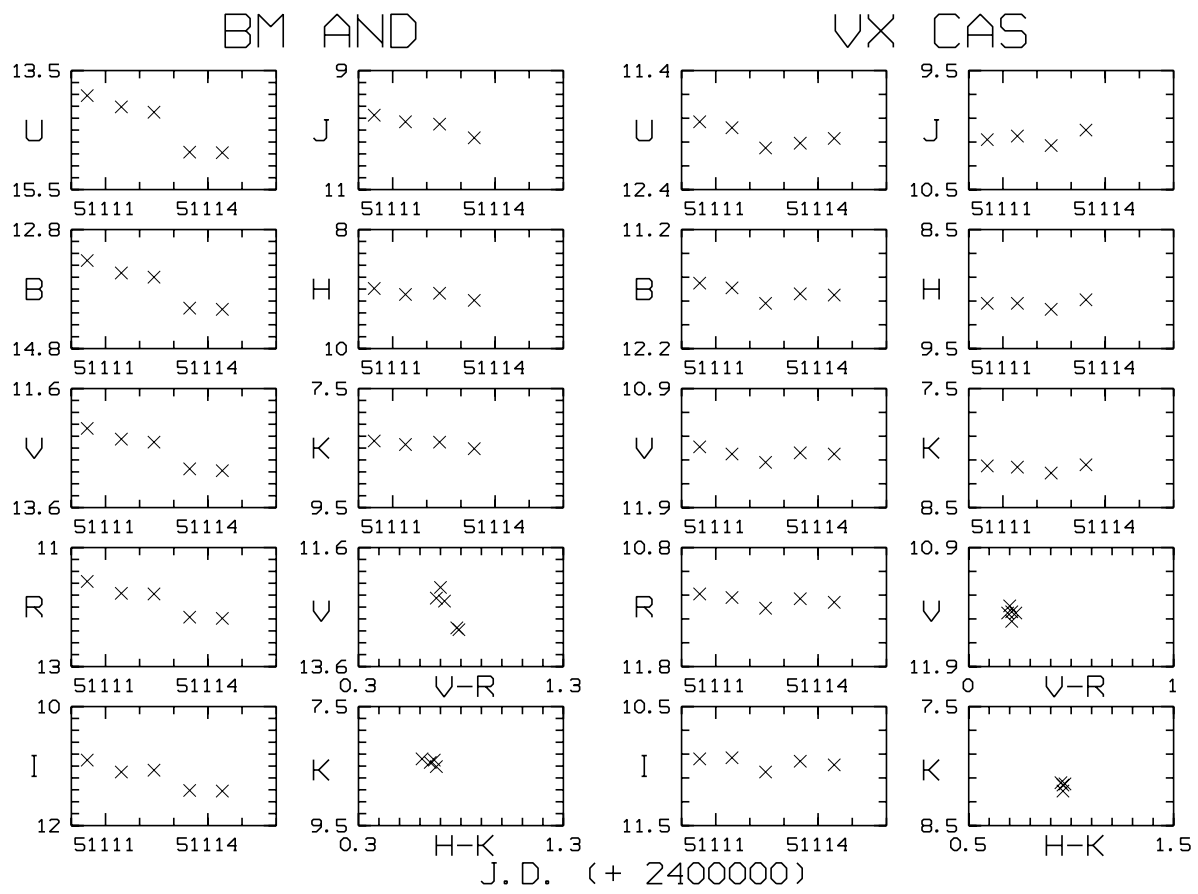

Fig. 1. Optical and near-IR light curves for BM And (left) and VX Cas (right) vs. Julian Date (+2 400 000). Magnitude errors are always smaller than the tickmarks in the magnitude axes $(0.2 \mathrm{mag})$. Color-magnitude diagrams of the data are also shown.

\section{CW Tau}
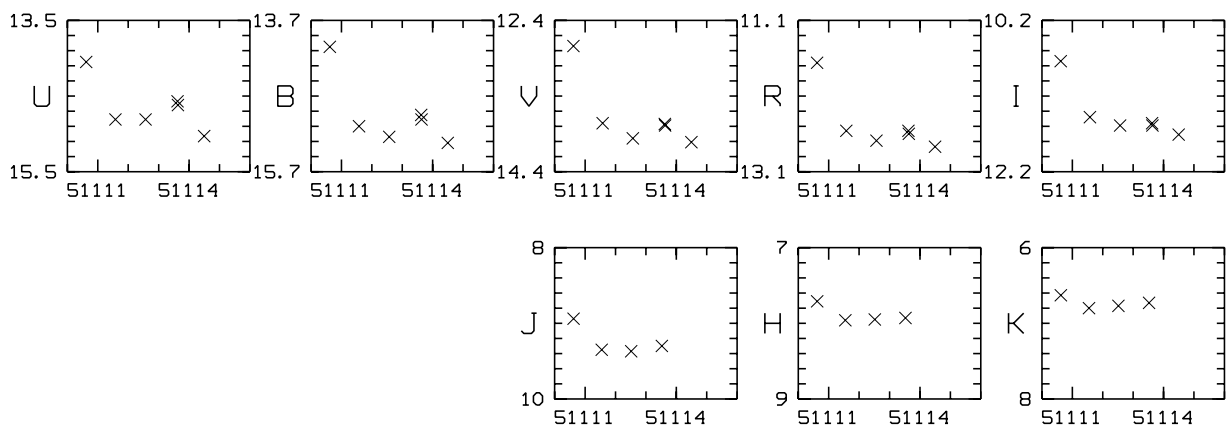

Julian Date $(+2400000)$
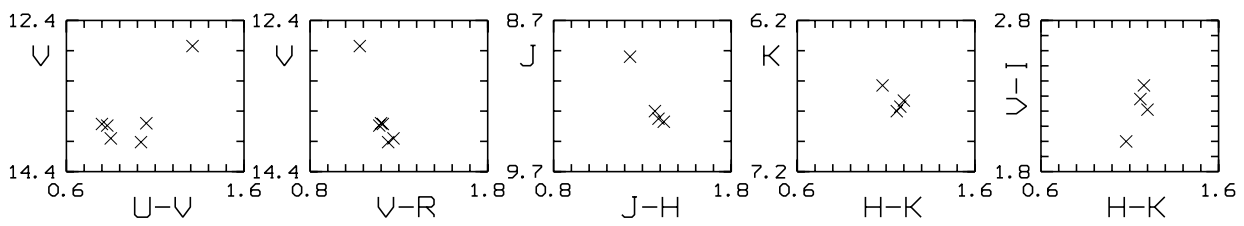

Fig. 2. Optical (top) and near-IR (middle) light curves for CW Tau vs. Julian date (+2400 000). Magnitude errors are always smaller than the tickmarks in the magnitude axes $(0.2 \mathrm{mag})$. Color-magnitudes diagrams are shown at the bottom. Also shown is the $H-K$ versus $V-I$ diagram (bottom-right).

practically constant while $U-V$ is bluer when the star is fainter. A single scenario could fit the variability from $V$ to $K$ (perhaps also including $B$ ). The results of Table 2 suggest that the observed near-IR variability amplitudes are clearly smaller than those expected from cold spots. Both hot and cold spots, as well as variable extinction, predict larger ultraviolet variability than observed. If we assume variable obscuration as the mechanism at work, the $U$ and $B$ flux excesses could reflect the contribution of scattered light, as suggested by Bouvier et al. (1995). On the other hand, ultraviolet excesses and veiling in CTTs are attributed to variable accretion from the gaseous disk (e.g. Hartigan et al. 1995). CW Tau has a noticeable veiling (Mora et al. 2001). EXPORT spectra reveal veiling values of $2.0 \pm 0.5$ (JD 2451113.585) and $1.3 \pm 0.5$ (JD 2451114.567); these spectra were obtained simultaneously to the last two optical measurements, when the star was close to its observed faintest state (Fig. 2). 
DR Tau
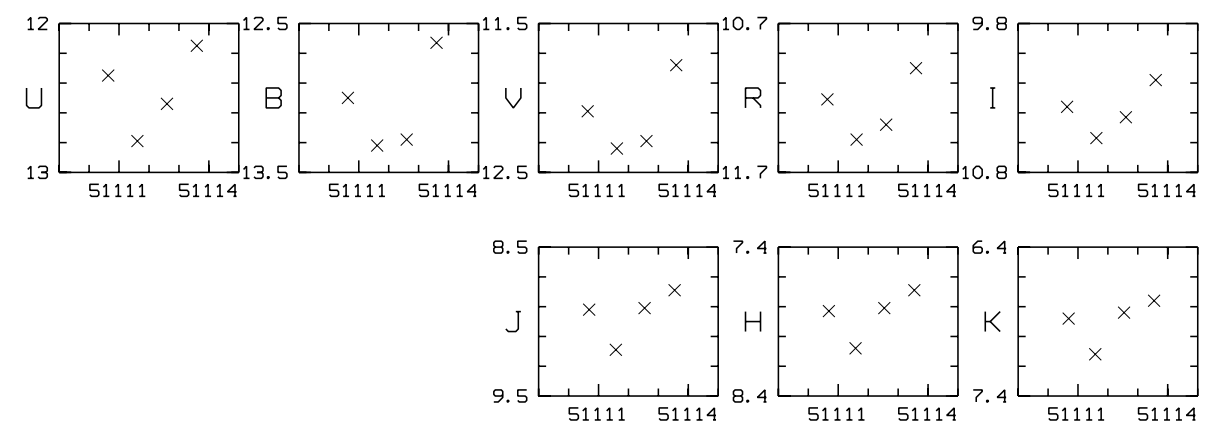

Julian Date $(+2400000)$

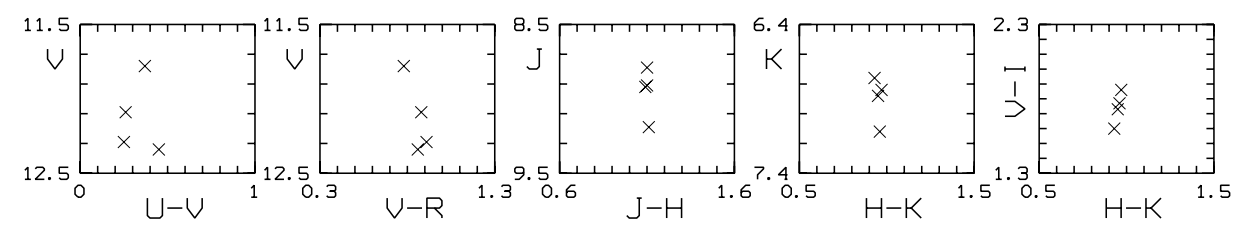

Fig. 3. As in Fig. 2 for DR Tau.

\section{RY Tau}
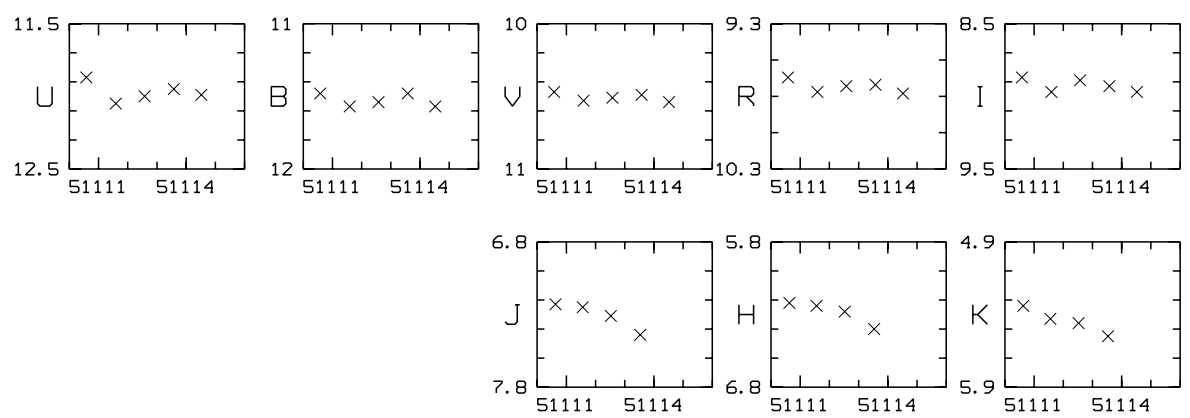

Jul lan Date $(+2400000)$
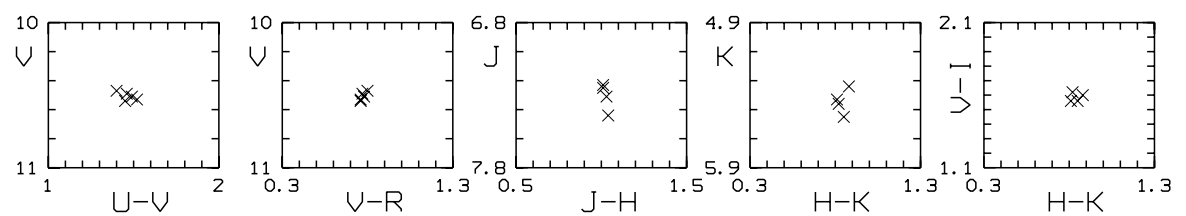

Fig. 4. As in Fig. 2 for RY Tau.

DR Tau was observed close to its average brightness level (Fig. 3). The amplitudes tend to decrease with wavelength, although this is not strictly true when day to day variations are considered; i.e. from JD 2451111.5 to $2451112.5 B$ and $V$ remained practically constant while $U R I$ and $J H K$ showed a considerable variability. DR Tau exhibits a remarkable $U$-excess, $U-B<-0.48$, and also veiling (Mora et al. 2001). The colors $B-V, V-R$ and $V-I$ tend to be redder when the star is fainter, the behaviour is more erratic in $U-V$ while $J-H$ and $H-K$ remain close to constant. Table 2 suggests that while the observed optical variability amplitudes could be fit by spots, the simultaneous near-IR amplitudes are larger than those predicted by such spots. This behaviour could be explained if an additional physical, variable process, likely related to the dusty disk, contributes to the near-IR variations.

\subsection{Stars with different optical and near-IR variability trends}

Here we discuss the objects for which the optical and nearIR variations do not follow the same trend.

RY Tau: RY Tau is known to change its mean light level and since October 1996, when the star experienced a brightening, its UXOR-type variability has been in the range $V \approx 9.5-11.0$ mag (Herbst \& Shevchenko 1999; Petrov et al. 1999). We observed the star at $V \sim 10.5 \mathrm{mag}$, close to the photometric minimum observed after the brightening. In the optical bands, small variations at the accuracy level of the individual measurements are present and follow the same trend (Fig. 4). On the other hand, the near-IR amplitudes are definitely larger than the optical ones and their behaviour as a function of time is different. 
DK Tau
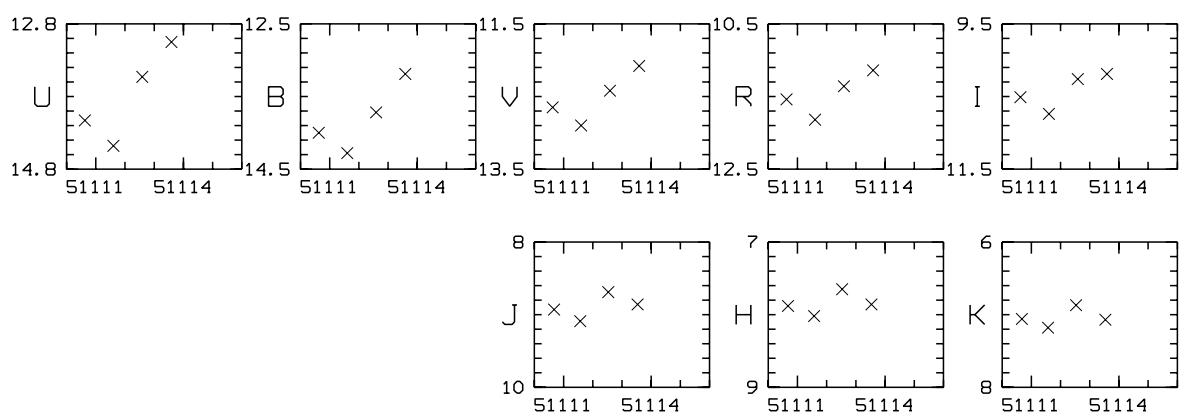

Jul lan Date $(+2400000)$
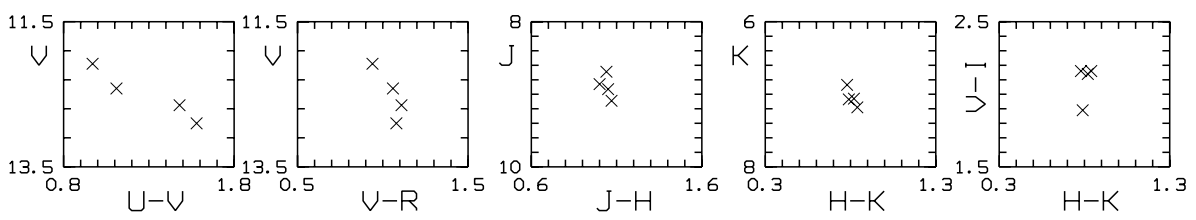

Fig. 5. As in Fig. 2 for DK Tau.

\section{UX Or 1}
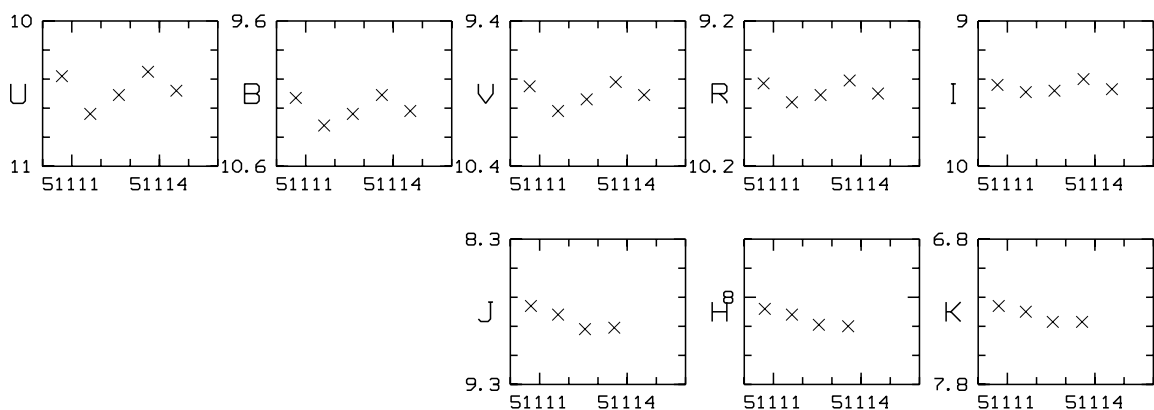

Julian Date (+ 2400000)
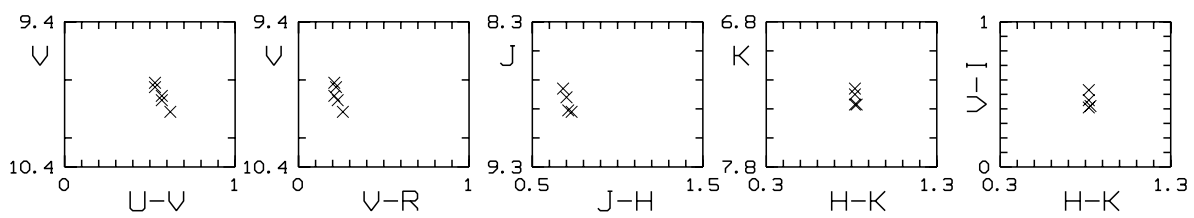

Fig. 6. As in Fig. 2 for UX Ori.

The data suggest that the mechanism responsible for the near-IR variability has no strong influence in the optical fluxes.

DK Tau: Its optical and near-IR variations follow the same trend from JD 2451110.5 to 2451112.5 , but from JD 2451112.5 to 2451113.5 the near-IR brightness decreases while the optical one increases (Fig. 5). As shown in Table 2 the optical and near-IR behaviour from JD 2451110.5 to 2451112.5 can be reasonably well explained in terms of spots or a nearly grey variable extinction (with the exception of the observed large $U$ amplitude). However, such simple scenarios do not account for the optical and near-IR variability trends observed from JD 2451112.5 to 2451113.5 . Cohen \& Schwartz (1976) also observed the same behaviour in DK Tau.

UX Ori: UX Ori is the prototype of type III variables, yet it does not always display the well known
UXOR photo-polarimetric anti-correlation (Oudmaijer et al. 2001). UX Ori was close to its maximum light level and its brightness variation was small (Fig. 6). However, the optical and near-IR trends are different. At first, UX Ori faded at UBVRI and JHK from JD 2451110.7 to JD 2451111.6, but contrary to the behaviour in the optical it continued to fade in $J H K$ till 2451113.6. Similar results are seen in the data by Hutchinson et al. (1994). Thus, distinct processes for the optical and near-IR variabilities are most likely playing a role.

CO Ori: CO Ori was observed in a relative light minimum well below its average magnitude, $\langle V\rangle=10.67$ (Herbst \& Shevchenko 1999). Amplitudes in the optical bands are $\approx 0.5 \mathrm{mag}$, with the colors remaining practically constant - a weak blueing in $U-B$ and $U-V$ could be present (Fig. 7). The near-IR variability decreases with wavelength and the star becomes redder when fainter. 


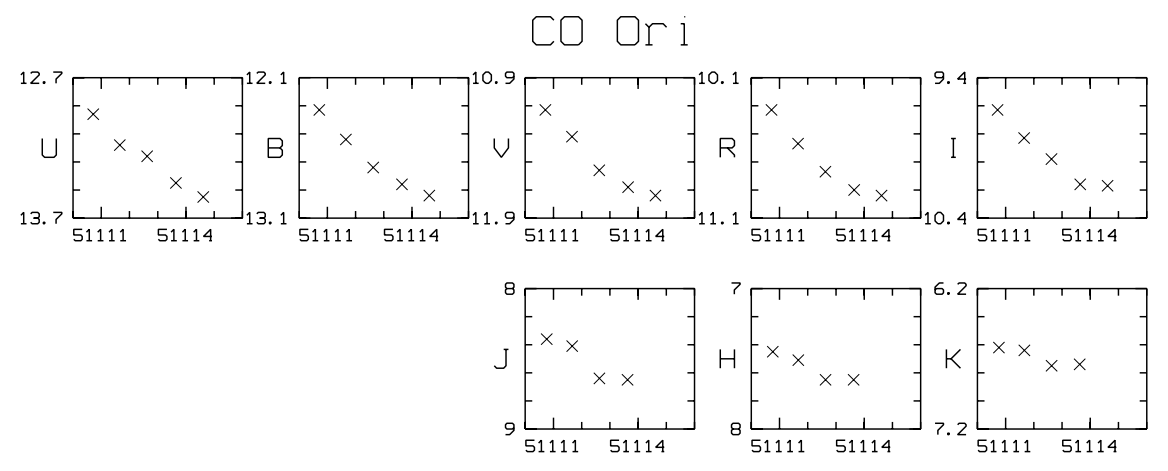

Julıan Date $(+2400000)$
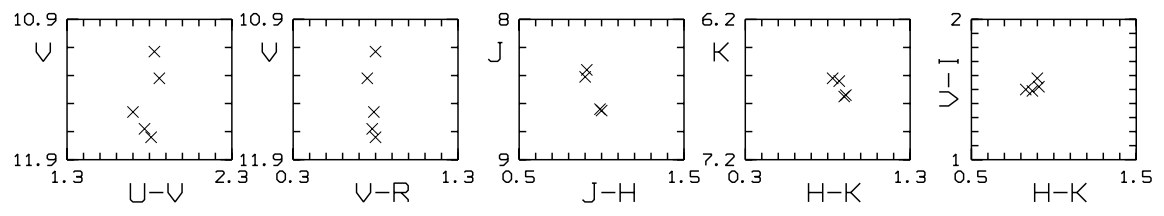

Fig. 7. As in Fig. 2 for CO Ori.

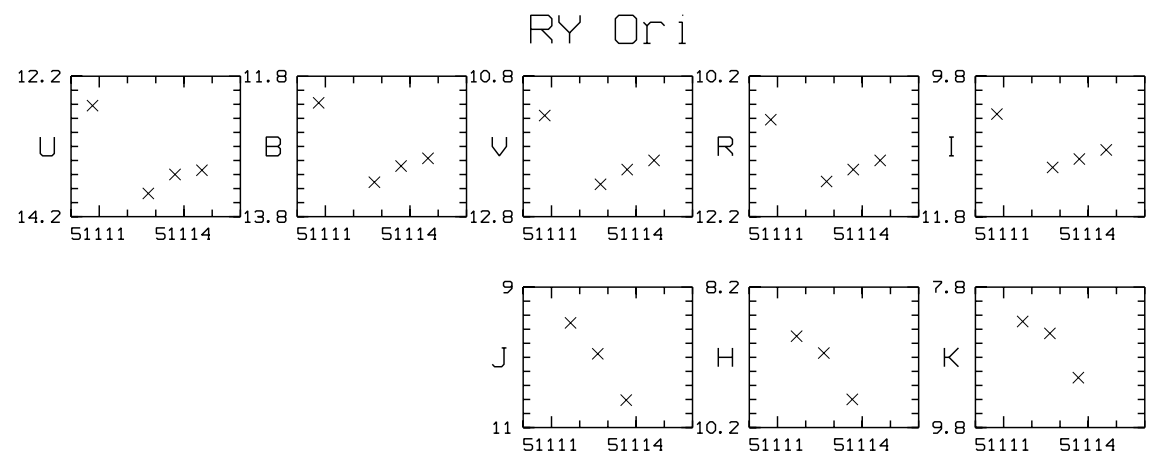

Julian Date( +2400000$)$
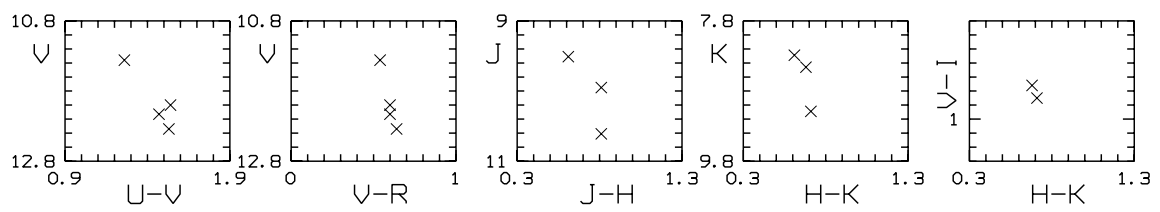

Fig. 8. As in Fig. 2 for RY Ori.

The fact that the optical variations are equally large in all bands is hard to explain in terms of spots; if we consider variable obscuration, dust grains should be considerably larger than typical interstellar grains in order to produce a grey extinction. Considering the optical and near-IR variations, we conclude that they most likely have different origins.

RY Ori: Strong variability is observed in all bands starting from a light level close to the maximum (Fig. 8). The trends, however, clearly differ in the optical and nearIR. The optical brightness decreases from JD 2451110.7 to JD 2451112.7, and from that date onwards it brightens again. The amplitudes decrease with wavelength. In the near-IR, RY Ori fades from JD 2451111.7 to JD 2451113.7. Thus, a single scenario is not able to explain the behaviour in both wavelength ranges.

CQ Tau: Figure 9 presents the light curves of CQ Tau, which was observed at maximum brightness but was also highly variable with $\Delta V \sim 0.5 \mathrm{mag}$. The variability patterns in the optical and near-IR are slightly different, suggesting different mechanisms could be at work. However, if we only consider the variability from JD 2451112.7 to 2451113.7 its behaviour is consistent with spots (Table 2). It is worth noting that the optical polarization did not anti-correlate with brightness and followed different trends in the $U B V$ and $R I$ bands (Oudmaijer et al. 2001).

\section{Discussion}

As described in the previous section, the observed variable stars can be gathered in two large groups, both with their own complexity: i) Stars whose optical and near-IR variations behave similarly over time. A relatively simple scenario can be drawn for most of these stars, such as spots or variable obscuration affecting the photospheric fluxes. In some stars, an ultraviolet excess $(U$ and $B)$ is observed, 
CQ Tau
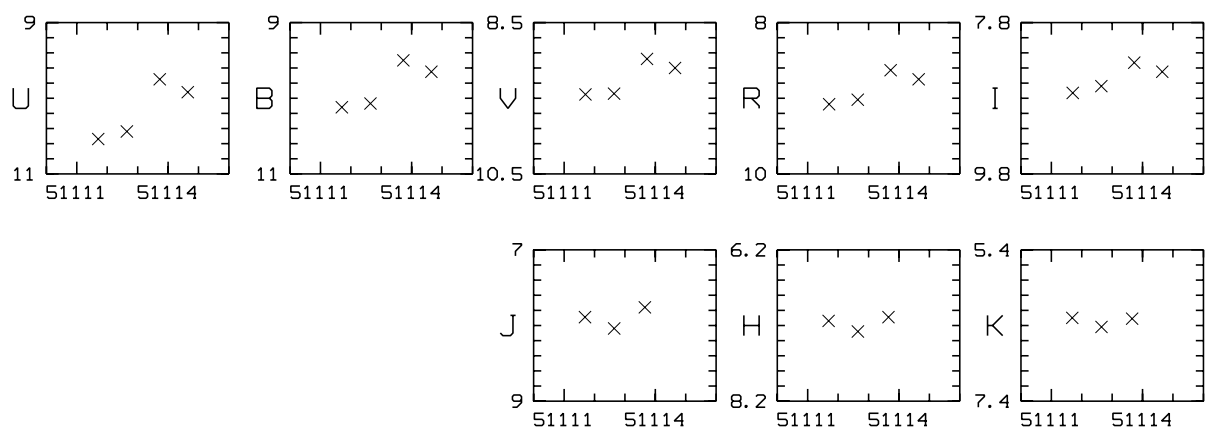

J. D. $(+2400000)$
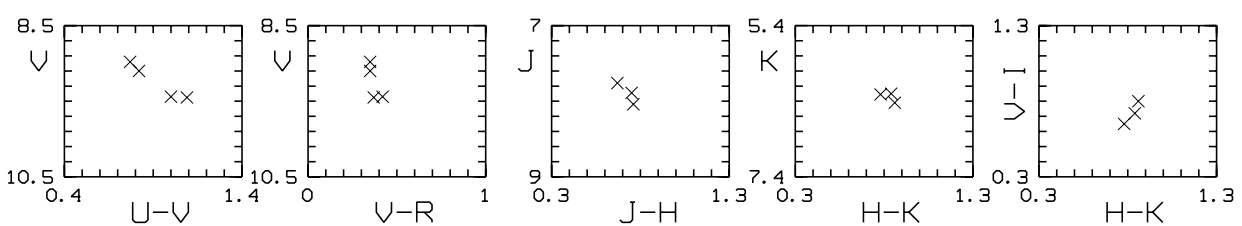

Fig. 9. As in Fig. 2 for CQ Tau.

which is probably due to the contribution of gas accretion from the disk or to scattered light. Furthermore, in some cases (DR Tau in our sample) the near-IR amplitudes suggest the presence of a variability mechanism in addition to spots or variable obscuration. ii) Stars whose optical and near-IR variabilities are uncorrelated. In these cases, there is no clear dependence of the variability on wavelength. These stars require a more complicated scenario to explain the optical variability on the one hand, and the near-IR variability on the other hand. It is worth noting that membership of either of the two groups is not dependent on variability amplitudes, object brightness level, the stellar spectral type (as indicated by the simultaneous spectroscopic observations of Mora et al. 2001), or to a correlation with optical polarization properties (Oudmaijer et al. 2001). In addition, a star can move in time from any of the groups to another, e.g. Cohen \& Schwarz (1976) observed an anti-correlation between the UBVRI and IR variabilities in BM And, while both are correlated in our data.

In general, optical fluxes, and consequently their variability, reflect the PMS photosphere and any physical processes affecting its emission. Near-IR fluxes are due to the photospheric emission plus a contribution from the circumstellar disk (Meyer et al. 1997; Natta et al. 2001), which implies that disk changes of any kind would influence the observed variations in this wavelength range. The near-IR variability is poorly understood and plausible scenarios must consider both the relative flux contribution from the PMS photosphere and the disk at any given time. A proper evaluation of both contributions would require many simultaneous observations and is not an easy task. We can provide, however, crude, approximate estimates of the near-IR fluxes coming from the disk in our sample of objects. These estimates can be made by comparing the observed mean $J H K$ magnitudes to the expected mean photospheric values. The latter can be estimated from the observed mean $V$ magnitude in October 98 and extrapolating it to $J H K$ using intrinsic colors of the corresponding stellar spectral types, taking into account a mean $A_{V}$ extinction calculated from the observed mean colors and an interstellar extinction law (e.g. Cohen et al. 1981). We assume intrinsic colors as given by Tokunaga (2000) for luminosity class $\mathrm{V}$ stars and the total (circumstellar+interstellar) $A_{V}$ is calculated from the observed mean $R-I$ color, chosen as the optimum one reflecting the PMS photospheres (e.g. Meyer et al. 1997). The mean expected photospheric JHK magnitudes and the estimated excesses at each near-IR band (differences between the expected photospheric magnitudes and the mean observed ones) are given in Table 3 , together with the mean observed $V$ magnitudes (Col. 2) and the estimated $A_{V}$ values (Col. 3). Similar $A_{V}$ values are found in the literature for our sample of stars. The expected $J H K$ photospheric magnitudes have been calculated using the expressions:

$$
\begin{aligned}
J_{\text {phot }} & =V_{\text {mean }}^{\text {Oct98 }}-(V-J)_{0}-0.735 A_{V} \\
H_{\text {phot }} & =V_{\text {mean }}^{\text {Oct98 }}-(V-H)_{0}-0.845 A_{V} \\
K_{\text {phot }} & =V_{\text {mean }}^{\text {Oct98 }}-(V-K)_{0}-0.910 A_{V} .
\end{aligned}
$$

The results in Table 3 show: i) The excesses are an estimate of the excess emission at the different wavelengths caused by the circumstellar disk. Thus, the increase of the excess emission from $J$ to $K$ means that the disk contribution increases with wavelength - as expected if thermal emission from dust dominates the disk fluxes. ii) In all cases, observed $K$-fluxes are dominated by disk emission, i.e. the $K$-flux from the disk contributes more than $50 \%$ of the observed flux (an excess of $0.75 \mathrm{mag}$ ). This is also often the case when $H$-fluxes are considered. iii) The stellar photosphere is the main contributor to the $J$-band emission, though in many cases the disk also contributes significantly and can even be at the same flux level as 
Table 3. Expected photospheric JHK magnitudes and estimated near-IR excesses (difference between the expected photospheric magnitude and the mean observed one) of variables stars during October 1998.

\begin{tabular}{lllllllll}
\hline Object & $V_{\text {mean }}^{\text {Oct98 }}$ & $A_{V}$ & $J_{\text {phot }}$ & $E_{\mathrm{J}}$ & $H_{\text {phot }}$ & $E_{\mathrm{H}}$ & $K_{\text {phot }}$ & $E_{\mathrm{K}}$ \\
\hline VX Cas & 11.5 & 1.4 & 10.4 & 0.4 & 10.3 & 1.2 & 10.2 & 2.0 \\
XY Per & 9.1 & 1.1 & 8.3 & 0.6 & 8.1 & 1.3 & 8.0 & 2.1 \\
CW Tau & 13.7 & 2.3 & 10.2 & 0.9 & 9.4 & 1.5 & 9.1 & 2.4 \\
RY Tau & 10.5 & 2.4 & 7.7 & 0.4 & 7.2 & 0.9 & 6.9 & 1.5 \\
DK Tau & 12.5 & 2.0 & 8.8 & -0.1 & 8.1 & 0.3 & 7.9 & 0.9 \\
DR Tau & 12.1 & 1.2 & 8.9 & 0.0 & 8.4 & 0.5 & 8.2 & 1.3 \\
UX Ori & 9.9 & 0.8 & 9.0 & 0.2 & 9.0 & 0.8 & 8.9 & 1.6 \\
CO Ori & 11.5 & 2.2 & 8.9 & 0.4 & 8.3 & 0.8 & 8.1 & 1.4 \\
HK Ori & 11.7 & 0.8 & 10.0 & 0.7 & 9.6 & 1.4 & 9.5 & 2.3 \\
RY Ori & 12.0 & 1.3 & 10.1 & 0.1 & 9.7 & 0.4 & 9.5 & 0.9 \\
CQ Tau & 9.2 & 0.4 & 8.1 & 0.2 & 7.9 & 0.7 & 7.8 & 1.5 \\
T Ori & 10.6 & 2.0 & 8.9 & 0.5 & 8.7 & 1.4 & 8.6 & 2.2 \\
BF Ori & 9.7 & 0.7 & 9.1 & 0.1 & 9.0 & 0.5 & 9.0 & 1.1 \\
RR Tau & 11.1 & 2.0 & 9.7 & 0.6 & 9.5 & 1.4 & 9.4 & 2.3 \\
V350 Ori & 11.8 & 2.1 & 10.2 & 0.2 & 10.0 & 0.8 & 9.8 & 1.5 \\
VV Ser & 11.9 & 3.5 & 9.3 & 0.5 & 8.9 & 1.4 & 8.7 & 2.3 \\
WW Vul & 10.8 & 1.4 & 9.7 & 0.4 & 9.5 & 1.1 & 9.4 & 1.9 \\
BM And & 12.6 & 0.4 & 10.0 & 0.1 & 9.5 & 0.5 & 9.4 & 1.0 \\
\hline
\end{tabular}

the star. These results demonstrate that any photospheric variation yields a very small variability amplitude in the observed near-IR magnitudes (which include star+disk), particularly in the $K$ magnitude, and also in $H$. Thus, we are confident in asserting that the near-IR variability in stars with near-IR excesses is primarily due to changes in the disk. These changes do not seem to be directly related to changes in the photosphere, as the color-color $\left(H_{-}\right.$ $K, V-I$ ) diagrams demonstrate (Fig. 2 to Fig. 9, bottomright); $V-I$ color changes can be taken as representative of photospheric changes while $H-K$ color changes can be considered as representative of changes in the disk. In a number of cases there is no clear correlation between the photospheric and the circumstellar disk changes. We have to be cautious, however, interpreting the color-color diagrams since in some stars the photosphere can partly influence the observed individual $H-K$ colors, as suggested by the excesses in Table 3; furthermore, the number of data points we have at present is small. Mean dust temperatures estimated from the $H$ and $K$ disk fluxes deduced from Table 3 are in the $\sim 1500 \mathrm{~K}$ range, which indicates that the near-IR fluxes arise predominantly from the inner hot disk, as would be expected. Thus, independent of their nature, the physical processes causing the near-IR variability of PMS stars affect the thermal emission of the inner disk; these processes do not seem to produce a remarkable change of the dust temperature since the $H-K$ colors do not vary significantly, at least during this period. In addition, we cannot exclude that changes of the near-IR light scattered off the disk could be an additional contributor to the near-IR variability. In this respect, it is worth noting that PMS stars show remarkable near-IR intrinsic polarization attributed to the dusty disks, e.g. Tamura \& Sato (1989), Casali (1995), Whitney et al. (1997), and also that there is direct evidence from near-IR images of circumstellar disks seen in reflected light (e.g. McCaughrean et al. 2000; Ménard et al. 2000).

An additional interesting aspect posed by the results in this work is related to the timescale for the near-IR variability. Slow changes in disk properties and structure can be the cause of the long term near-IR variability; viscous timescale (months to years) can alter the accretion rate through the disk (Pringle 1981; Skrutskie et al. 1996); other mechanisms such as thermal instabilities, variable magnetic fields or warping instability can lead to variations on shorter timescales, several days, weeks (Carpenter et al. 2001, and references therein). It is, however, unclear, which physical mechanisms in the inner regions of the circumstellar disk are able to produce fluctuations of the near-IR fluxes of as much as 1 mag on time scales of 12 days, and probably shorter. This issue deserves further, theoretical and modeling investigations.

\section{Concluding remarks}

In this paper, we have analysed the simultaneous optical and near-IR variability behaviour of $18 \mathrm{HAeBe}$ and CTTs stars, most of them known to belong to the group of UXORs. Roughly speaking, the stars can be classified in two groups, one showing a correlation of the optical and near-IR fluctuations, i.e. they both become brighter or fainter simultaneously, and a second group in which this is not the case. The correlation (or lack thereof) in our data is apparently not related to any other observational 
property, such as spectral type, brightness level, variability amplitude, etc. In addition, a star is likely to be classified into either of the two groups depending on the epoch of the observations (and ultimately depending on the relative contribution of the circumstellar disks to the near-IR fluxes). The variability of the stars with the same trend in the optical and near-IR is probably mainly caused by a mechanism affecting the photospheric fluxes in both wavelength regimes; though in some cases the near-IR variability can also reflect structural disk variations. On the other hand, the objects with different simultaneous optical and near-IR trends unambiguously show photospheric variations and structural disk variations. Such structural disk changes are remarkable in the sense that they substantially affect the disk thermal emission and/or the amount of disk scattered light on short timescales. Changes of up to around $1 \mathrm{mag}$ in the near-IR on timescales of $1-2$ days are seen in our dataset. Optical versus near-IR colorcolor diagrams, e.g. $(H-K, V-I)$, suggest that dust disk changes are not directly related to photospheric changes. In summary, we feel that these simultaneous data reveal a need for: 1) theoretical and modeling work, investigating PMS circumstellar disk scenarios, to shed light on plausible mechanisms able to explain the observational results, 2) new simultaneous optical and near-IR observations with a better time coverage than those presented here. Such observations would make it possible to soundly disentangle the PMS photospheric emission and its variability from the disk emission and its variability, which must constitute a basic goal of future theoretical work in this field.

Acknowledgements. We appreciate the discussions and collaboration provided by M. Fernández concerning starspot models. A. Alberdi, C. Eiroa, B. Merín, B. Montesinos, A. Mora, J. Palacios and E. Solano are supported in part by Spanish grants ESP 98-1339 and AYA 2001-1124.

\section{References}

Appenzeller, I., \& Mundt, R. 1989, A\&ARv, 1, 291

Bertout, C. 2000, A\&A, 363, 984

Beust, H., Karmann, C., \& Lagrange, A.-M. 2001, A\&A, 366, 945

Bouvier, J., Covino, E., Kovo, O., et al. 1995, A\&A, 299, 89

Bouvier, T., Chelli, A., Allain, S., et al. 1999, A\&A, 349, 619

Carpenter, J. M., Hillenbrand, L. A., \& Skrutskie, M. F. 2001, AJ, 121,3160

Casali, M. M. 1995, MNRAS, 277, 1385

Chelli, A., Carrasco, L., Mujica, R., et al. 1999, A\&A, 345, L9

Cohen, J. G., Frogel, J. A., Persson, S. E., \& Elias, J. H. 1981, ApJ, 249, 481

Cohen, M., \& Schwartz, R. D. 1976, MNRAS, 174, 137

Davies, J. K., Evans, A., Bode, M. F., \& Whittet, D. C. B. 1990, MNRAS, 247, 517

de Winter, D., van den Ancker, M. E., Thé, P. S., et al. 2001, A\&A, 380, 609
Eiroa, C., Mora, A., Palacios, J., et al. (EXPORT) 2000, in Disks, Planetesimals and Planets, ed. F. Garzón, C. Eiroa, D. de Winter, \& T. Mahoney, ASP Conf. Ser., 219, 3

Eiroa, C., Garzón, F., Alberdi, A., et al. (EXPORT) 2001, A\&A, 365, 110

Evans, A., Davies, J. K., Kilkenny, D., \& Bode, M. J. 1989, MNRAS, 237, 695

Fernández, M., \& Eiroa, C. 1996, A\&A, 310, 143

Gahm, G. F., Fischerström, C., Liseau, R., \& Lindroos, K. P. 1989, A\&A, 211, 115

Grady, C. A., Sitko, M. L., Russel, R. W., et al. 2000, in Protostars and Planets IV, ed. V. Mannings, A. P. Boss, \& S. S. Russell (Tucson: University of Arizona Press), 613

Grinin, V. P. 2000, in Disks, Planetesimals and Planets, ed. F. Garzón, C. Eiroa, D. de Winter, \& T. Mahoney, ASP Conf. Ser., 219, 216

Grinin, V. P., Kolotilov, E. A., \& Rostopchina, A. 1995, A\&AS, 112,457

Hartigan, P., Edwards, S., \& Ghandour, L. 1995, ApJ, 452, 736

Hartmann, L., Kenyon, S. J., \& Calvet, N. 1993, ApJ, 407, 219

Herbst, W., Herbst, D. K., Grossman, E. J., \& Weinstein, D. 1994, AJ, 108, 1096

Herbst, W., \& Shevchenko, V. S. 1999, AJ, 118, 1043

Hutchinson, M. G., Albinson, J. S., Barrett, P., et al. 1994, A\&A, 285, 863

Hutchinson, M. G., Evans, A., Davies, J. K., \& Bode, M. F. 1989, MNRAS, 237, 683

McCaughrean, M. J., Stapelfeldt, K. R., \& Close, L. M. 2000, in Protostars and Planets IV, ed. V. Mannings, A. P. Boss, \& S. S. Russell (Tucson: University of Arizona Press), 485

Ménard, F., Stapelfelt, K., Duchêne, G., et al. 2000, in Disks, Planetesimals and Planets, ed. F. Garzón, C. Eiroa, D. de Winter, \& T. Mahoney, ASP Conf. Ser., 219, 140

Meyer, M. R., Calvet, N., \& Hillenbrand, L. A. 1997, AJ, 114, 288

Mora, A., Merín, B., Solano, E., et al. (EXPORT) 2001, A\&A, 378,116

Natta, A., Grinin, V. P., \& Tambovtseva, L. V. 2000, ApJ, 542, 421

Natta, A., Prusti, T., Neri, R., et al. 2001, A\&A, 371, 186

Natta, A., \& Whitney, B. A. 2000, A\&A, 364, 633

Oudmaijer, R. D., Palacios, J., Eiroa, C., et al. (EXPORT) 2001, A\&A, 379, 564

Petrov, P. P., Zajtseva, G. V., Efimow, Yu. S., et al. 1999, A\&A, 341, 553

Pringle, J. E. 1981, ARA\&A, 19, 137

Rydgren, A. E., \& Vrba, F. J. 1983, AJ, 88, 1017

Shevchenko, V. S., Grankin, K. N., Ibragimov, M. A., Melnikov, S. Yu., \& Yakubov, D. 1993, Ap\&SS, 202, 121

Skrutskie, M. F., Meyer, M. R., Whalen, D., \& Hamilton, C. 1996, AJ, 112, 2168

Thé, P. S., de Winter, D., \& Pérez, M. R. 1994, A\&AS, 104, 315

Tamura, M., \& Sato, S. 1989, AJ, 98, 1368

Tokunaga, A. T. 2000, in Allen's Astrophysical Quantities, ed. A. N. Cox (Springer), 143

Walker, M. F. 1980, PASP, 92, 66

Whitney, B. A., Kenyon, S. J., \& Gómez, M. 1997, ApJ, 485, 703 